\title{
DEEP BINARY REPRESENTATION OF FACIAL EXPRESSIONS: A NOVEL FRAMEWORK FOR AUTOMATIC PAIN INTENSITY RECOGNITION
}

\author{
Mohammad Tavakolian and Abdenour Hadid
}

\author{
Center for Machine Vision and Signal Analysis (CMVS), University of Oulu, Finland
}

\begin{abstract}
Automatic pain assessment is crucial in clinical diagnosis. Experiencing pain causes deformations in the facial structure resulting in different spontaneous facial expressions. In this paper, we aim to represent the facial expressions as a compact binary code for classification of different pain intensity levels. We divide a given face video into non-overlapping equal-length segments. Using a Convolutional Neural Network (CNN), we extract features from randomly sampled frames from all segments. The obtained features are aggregated by exploiting statistics to incorporate low-level visual patterns and high-level structural information. Finally, this processed information is encoded using a deep network to obtain a single binary code such that videos with the same pain intensity level have smaller Hamming distance than those of different levels. Extensive experiments on the publicly available UNBC-McMaster database demonstrates that our proposed method achieves superior performance compared to the state-of-the-art.
\end{abstract}

Index Terms - Pain Assessment, Facial Expression Analysis, Deep Learning, Binary Representation, Clinical Diagnosis

\section{INTRODUCTION}

Pain assessment is an important part of many clinical diagnosis applications. The pain level of a patient is routinely evaluated using standard pain assessment methods, e.g. the Visual Analogue Scale (VAS) [1] and the Numeric Rating Scale (NRS) [2]. However, these methods mainly rely on the patient's self-report. Therefore, the use of these methods is very limited especially with people who are not able to communicate their experiences such as neonates and unconscious patients (e.g. patients after surgical operation). In such cases, pain assessment can also be done by manually monitoring the behavioral and physiological changes in the patient. However, clinicians may provide a subjective assessment that can vary among the practitioners and be affected by the personal behavioral characteristics of the patient. In addition, manual pain assessments do not allow for continuous monitoring of patients.

To overcome the aforementioned limitations, machine learning and computer vision techniques have been proposed as appealing alternatives for automatic pain assessment, aiming at objective and efficient pain estimation. Earlier works focused on binary pain recognition, i.e. detecting pain versus no pain. Later on, researchers started looking at continuousvalue pain assessment that is more useful for clinical analysis. Among automatic pain assessement methods, analyzing facial expressions has received a considerable attention, since the human face can be considered as a mirror of the health conditions.

Works on automatic pain recognition from facial expressions are mostly based on the Prkachin and Solomon's Pain Intensity metric (PSPI) [3] which measures pain as a linear combination of facial action units (FAU). Facial action units refer to the movements of specific facial muscles involved in facial expressions. Earlier works considered pain recognition as a binary classification problem [4, 5], i.e. distinguishing pain from no pain. However, pain has different levels of intensities. Kaltwang et al. [6] divided the facial images into a uniform grid of cells and extracted Local Binary Pattern (LBP) features. They used these data as appearance features for pain estimation. Florea et al. [7] proposed a histogram of topographical features for pain estimation in a transfer learning framework. Exploiting the temporal progression of pain expression from neutral through the apex and then back to neural, Zhao et al. [8] proposed ordinal information for pain estimation. More recently, a handful of deep learning based methods have been applied to the problem of pain estimation. For instance, Zhou et al. [9] proposed a Recurrent Convolutional Neural Network (RCNN) which uses a time-windowed to capture features extracted from flattened frames. Egde et al. [10], in another work, encoded the temporal information using a deep network and fused the information with handcrafted features.

A crucial step towards robust automatic pain recognition is the extraction of the most discriminative information from the facial expressions. This is a challenging task due to the fact that facial expressions exhibit complex low-level visual patterns in each frame of the video as well as high-level spatiotemporal information across the entire length of the video. In this paper, we aim to classify different levels of pain in a given video by tracking the spontaneous facial expression variations and encoding them into a fixed-length binary code.

We devise a novel deep architecture to extract the promi- 
nent features from the videos into a binary code. The videos are encoded so that the distances between the binary codes of the same pain levels are much smaller than the distances of pains of different levels. This encoding strategy maximizes the inter-level distances while minimizing the intra-level distances.

Among our contributions in this paper, we can cite: First, we propose an end-to-end deep architecture for binary representation of different pain intensity levels that is independent from the length of the video. Second, we incorporate features from both low-level visual patterns and high-level structures of the facial videos. Finally, we perform extensive experiments on a publicly available database demonstrating the efficiency of the proposed method in classifiying different levels of pain.

\section{DEEP BINARY REPRESENTATION}

Suffering from pain results in structural deformations shown in the face. Exploiting the intensities of these facial expression variations allows representing different pain intensity levels. The pain intensity representation should be sensitive to instantaneous changes in the appearance of the face regardless of the identity of the subject. This ensures keeping track of subtle changes in the facial structure and mapping these changes into an appropriate pain intensity level. To obtain such a representation, we propose a deep architecture that extracts features from randomly sampled frames of the given video using a Convolutional Neural Network (CNN). These features are fed into an aggregation layer to capture their distribution and acquire a video-level representation. Then, the video-level representation is passed through a deep binary encoding network, which is devised based on Multi-Layer Perceptron (MLP), to encode the intermediate features into a single binary code. Finally, the classification is done by measuring the Hamming distance between the binary codes of the query video and each pain intensity level of the training data, i.e. same pain intensity level is considered for two videos with the smallest Hamming distance. Figure 1 shows an overview of the proposed architecture for pain intensity recognition.

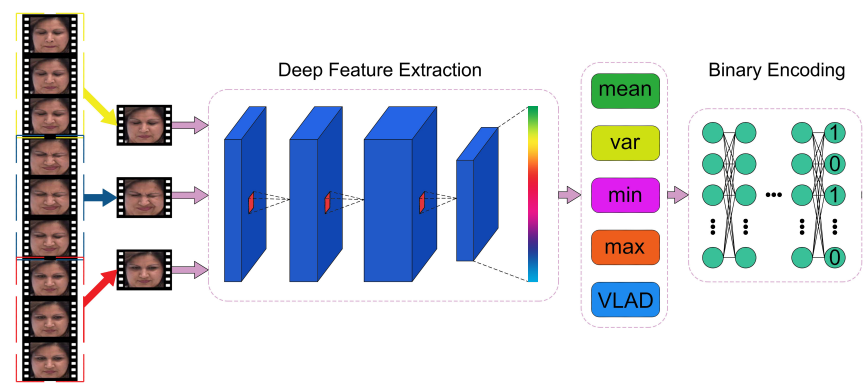

Fig. 1. An overview of the proposed deep binary representation architecture for robust automatic pain intensity recognition.

\subsection{Feature Extraction}

We partition a given video into $K$ non-overlapping segments $\left\{S_{1}, S_{2},, S_{K}\right\}$ of equal length, where $S_{i}$ denotes the $i$-th segment of the video. We randomly sample one frame $X_{i}$ from each segment as a snippet of the video. To capture the features from these data, we input the extracted snippets into a CNN and obtain a feature vector from the last fully connected layer of the network.

Moreover, we derive statistics from the obtained features that provide a better insight about the facial appearance variations in the entire video. We compute the average, variance, minimum, and maximum of features for each dimension. The average of features yields in the approximated location of data in the feature space and the variance reveals the degree of variations. Moreover, the minimum and the maximum values of features indicate the lower and the upper boundaries of the feature space.

To increase the discriminative power, we map the obtained features from the CNN into a high-dimensional space using Vector of Locally Aggregated Descriptor (VLAD) [11]. We exploit the CNN features to build a dictionary for VLAD representation. Given the deep features extracted from the CNN, K-mean clustering algorithm is applied to compute the cluster centroids for estimation of VLAD's components $v_{k, j}$.

$$
v_{k, j}=\sum x_{i, j}-c_{k, j}
$$

where $x_{i, j}$ is the $j$-th component of the CNN feature from the $i$-th snippet, and $c_{k, j}$ refers to the $k$-th cluster centroid. We perform $\ell_{2}$ optimization to obtain the VLAD representation. We compute the output of the aggregation layer for the set of video's snippets $X=\left\{X_{1}, X_{2}, \ldots, X_{K}\right\}$ as $F_{X}=$ $[\operatorname{mean}(X), \operatorname{var}(X), \min (X), \max (X), \operatorname{VLAD}(X)]^{T}$. Therefore, the aggregation layer increases the discriminative power of the extracted features for an effective binary representation.

\subsection{Video Binary Representation}

We aim to learn a mapping $b(x)$ that projects $p$-dimensional real-valued inputs $x \in R^{p}$ onto $q$-dimensional binary codes $h \in\{0,1\}^{q}$, while preserving notions of similarity. This mapping is achieved by a network of fully connected layers that is parametrized by $\theta^{(i)}=\left\{W^{(i)}, b^{(i)}\right\}$, where $W^{(i)}$ and $b^{(i)}$ are the weight matrix and the bias vector of the $i$-th layer, respectively. So, the entire network's parameters are defined as $\Theta=\left\{\theta^{(1)}, \theta^{(2)}, \ldots, \theta^{(N)}\right\}$. We choose the ReLu function as the non-linearity between layers and the last layer's activation is a sign function to generate a binary code. So, the compact representation of the mapping $b(x)$ is obtained as:

$$
b(x)=\operatorname{sign}(\Phi(x \mid \Theta))
$$

where $\operatorname{sign}(\cdot)$ denotes the element-wise sign function, and $\Phi(x \mid \Theta): R^{p} \rightarrow R^{q}$ is a real-valued transformation. 
The choice of loss function is crucial for learning appropriate similarity measures. The majority of existing binary coding techniques (e.g. [12, 13]) formulate learning objectives in terms of pairwise similarity, where pairs of inputs are labeled as either similar or dissimilar. However, it is nontrivial to label input pairs as similar or dissimilar in the context of pain intensity level. One way to avoid this problem is to define loss in terms of relative similarity. To define relative similarity, we assume that the training data includes triplets of snippet sets $\left\{X_{A}, X_{P}, X_{N}\right\}$ with the corresponding features $F_{X_{A}}, F_{X_{P}}$, and $F_{X_{N}}$. In terms of relative similarity, the pair $\left(F_{X_{A}}, F_{X_{P}}\right)$ is more similar than the pair $\left(F_{X_{A}}, F_{X_{N}}\right)$. The task is to learn a binary mapping function $b$ such that $b\left(F_{X_{A}}\right)$ is closer to $b\left(F_{X_{P}}\right)$ than to $b\left(F_{X_{N}}\right)$ in terms of Hamming distance. We use a ranking loss on the triplet of binary codes $\left\{h_{A}, h_{P}, h_{N}\right\}$ obtained from $b$ applied to $\left\{F_{X_{A}}, F_{X_{P}}, F_{X_{N}}\right\}$.

$$
\begin{aligned}
& L_{\text {triplet }}\left(h_{A}, h_{P}, h_{N}\right)= \\
& \quad \max \left(0,\left\|h_{A}-h_{P}\right\|_{H}-\left\|h_{A}-h_{N}\right\|_{H}+1\right)
\end{aligned}
$$

where $\|\cdot\|_{H}$ is the Hamming distance. This loss is zero when the Hamming distance between the more-similar pair, $\| h_{A}-$ $h_{P} \|_{H}$, is at least one bit smaller than the Hamming distance between the less-similar pair, $\left\|h_{A}-h_{N}\right\|_{H}$. Therefore, this loss function is more flexible as it can be used to preserve rankings among similar samples.

Given a training set of triplets, $\left\{\left(X_{A_{i}}\right),\left(X_{P_{i}}\right),\left(X_{N_{i}}\right)\right\}_{i=1}^{M}$, we define an objective function as the sum of the empirical losses and a regularization penalty on the sum of network's parameters.

$$
\begin{gathered}
J(\Theta)=\sum_{i=1}^{M} L_{\text {triplet }}\left(h_{A_{i}}, h_{P_{i}}, h_{N_{i}}\right)+ \\
\lambda \sum_{j=1}^{N}\left\|W^{(j)}\right\|_{F}^{2}+\left\|b^{(j)}\right\|_{2}^{2}
\end{gathered}
$$

However, this objective function is discontinuous and non-convex that make the optimization challenging. Although we cannot overcome the non-convexity, the discontinuity is tackled by defining a continuous upper-bound on the loss. The upper-bound on the loss that we exploit for learning the encoding function is defined as:

$$
\begin{gathered}
L_{\text {triplet }}\left(h_{A}, h_{p}, h_{N}\right) \leq \max _{g_{A}, g_{P}, g_{N}}\left\{L_{\text {triplet }}\left(g_{A}, g_{p}, g_{N}\right)+\right. \\
\left.g_{A}^{T} f\left(X_{A} \mid \Theta\right)+g_{P}^{T} f\left(X_{P} \mid \Theta\right)+g_{N}^{T} f\left(X_{N} \mid \Theta\right)\right\}- \\
\max _{h_{A}}\left\{h_{A}^{T} f\left(h_{A} \mid \Theta\right)\right\}-\max _{h_{P}}\left\{h_{P}^{T} f\left(h_{P} \mid \Theta\right)\right\}- \\
\max _{h_{N}}\left\{h_{N}^{T} f\left(h_{N} \mid \Theta\right)\right\}
\end{gathered}
$$

where $g_{A}, g_{P}, g_{N}, h_{A}, h_{P}$, and $h_{N}$ are constrained to be $q$-dimensional binary vectors. Summing the upper-bound instead of the loss in Eq. 4yyields a maximum limit on the regularized empirical loss. The resulting bound is continuous and

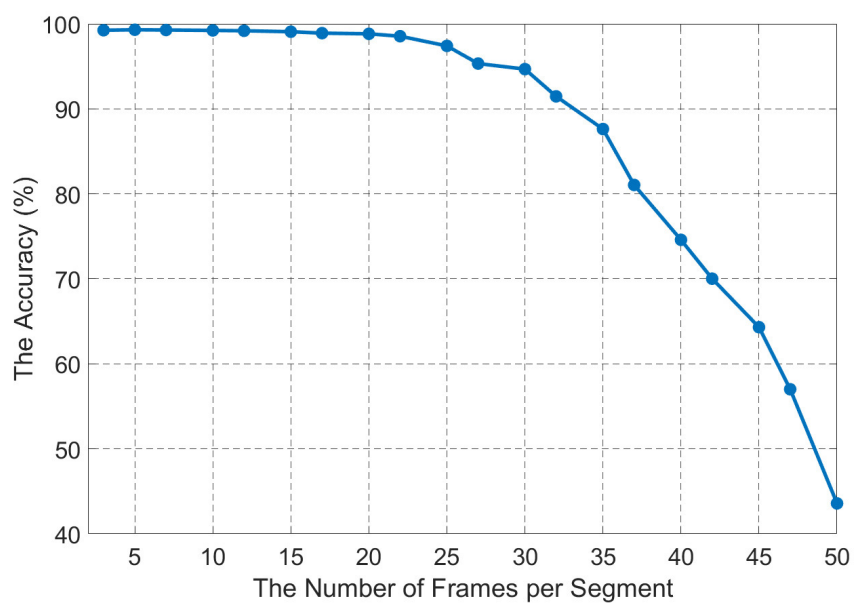

Fig. 2. The pain intensity recognition accuracy versus number of frames per segment.

piecewise smooth as long as $f$ is continuous in $\Theta$. In addition, the bound allows to learn encoding function based on non-linear functions $f$, e.g. ReLu.

\section{EXPERIMENTAL ANALYSIS}

We conducted experiments on the UNBC-McMaster Shoulder Pain Expression database [14] to evaluate the performance of our proposed method. We followed the standard protocol as in [14], i.e. leave-one-subject-out cross validation is used to avoid mixing the problem of pain intensity recognition with the subject identification.

We used a pre-trained CASIA face model [15] for the feature extraction step. The training is performed by making 3,000 triplets in each epoch from the training videos and the network is trained for 200 epochs.

Two hyper-parameters that directly affect the performance of the proposed method are the segments length and the feature combination. To get a better insight into the effect of these hyper-parameters, we performed a series of experiments to evaluate the performance of our proposed method under the variations of these factors. To assess the effect of different segments lengths, we partitionned the videos into segments of various lengths ranging from 3 to 50 frames per segment. The pain intensity recognition accuracy of our proposed method is depicted in Figure 2. It can be seen that the accuracy is quite steady until the range of 20 frames per segment. This further confirms that the redundancy between adjacent frames does not provide any additional information. However, the accuracy drops significantly as the length of segments increases, which indicates that loosing substantial temporal information between frames (as the result of random sampling) dwindles the accuracy of the deep model.

Furthermore, we analyzed the performance of the proposed method with different combinations of the features obtained from CNN. Using all the statistics (mean, variance, 

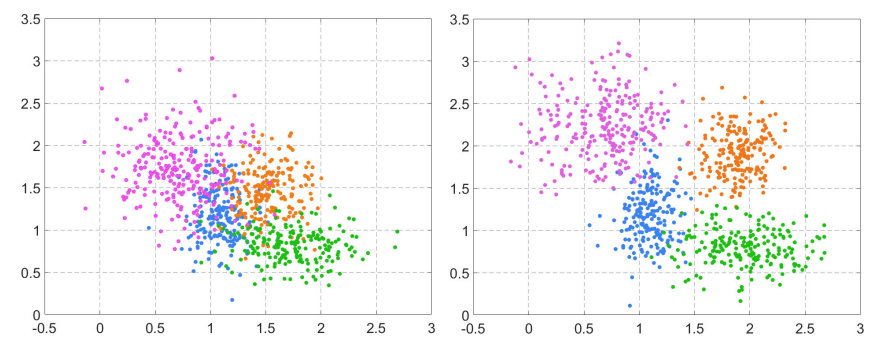

Fig. 3. Classification of four pain levels; Left: Using raw features extracted from CNN, Right: After applying statistics and VLAD.

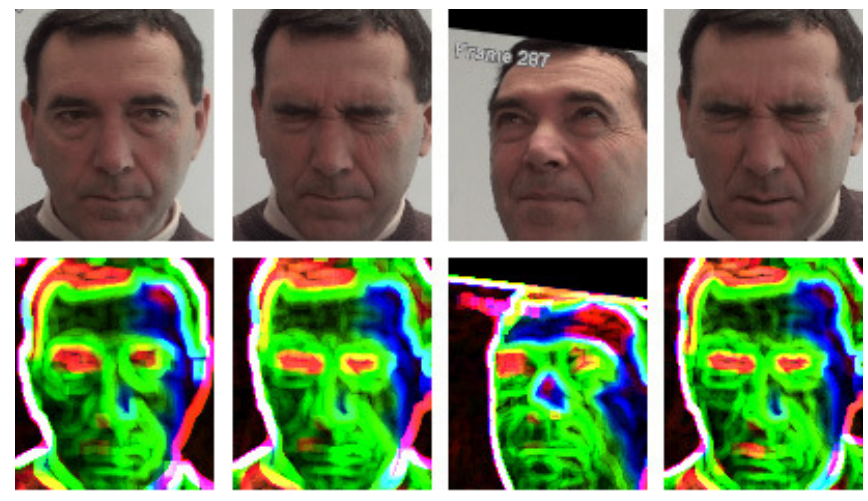

Fig. 4. Sample frames from the UNBC-McMaster database [14]; Top: The cropped faces, Bottom: The reconstructed patterns.

min, and max), our model achieves $98.54 \%$ accuracy in pain intensity recognition. However, combining the statistics of the features with the VLAD, the accuracy rises to $99.31 \%$. In addition, Figure 3 demonstrates the difference between the classification of samples from four pain levels when raw CNN features are used for binary representation versus the combination of the statistics and VLAD. The visualization of features shows that applying the statistics and VLAD increases the discriminative power of the extracted features from CNN.

The UNBC-McMaster Shoulder Pain Expression database [14] is widely used for pain expression recognition. This database contains facial videos of individuals performing a series of active and passive range-of-motion tests to their either affected or unaffected limbs in two sessions. Figure 4 (top) shows some samples from this database. Each video was annotated in a frame-level fashion, resulting in 16 discrete pain intensity levels $(0-15)$ based on facial action units. In our experiments, we used the active tests set that includes 200 videos of 25 subjects with 48,398 frames of the size $320 \times 240$ pixels.

Figure 4 (bottom) shows the reconstructed patterns from the sampled frames that cause high activations in a given feature maps. The regions of the face, such as nose, lip corners,
Table 1. Comparison of MSE and PCC on the UNBCMcMaster database [14].

\begin{tabular}{lcc}
\hline & MSE & PCC \\
\hline Kaltwang et al. $[16]$ & 1.39 & 0.59 \\
\hline Florea et al. $[7]$ & 1.21 & 0.53 \\
\hline Zhou et al. $[9]$ & 1.54 & 0.64 \\
\hline Zhao et al. $[8]$ & N/A & 0.60 \\
\hline Rodriguez et al. $[17]$ & 0.74 & 0.78 \\
\hline Our Proposed Method & $\mathbf{0 . 6 9}$ & $\mathbf{0 . 8 1}$ \\
\hline
\end{tabular}

lids, and brow, are highlighted as they are mostly affected by pain. These reconstructed patterns reveal the effectiveness of our proposed method in representating pain expressions from facial videos. To make a direct and fair comparison with the state-of-the-art approaches, we report Pearson Correlation Coefficient (PCC) and Mean Squared Error (MSE) in Table 1. As can be seen, our proposed method improves the performance of automatic pain intensity recognition by outperforming the existing benchmark approaches. We argue that the low MSE is due to incorporating low-level visual patterns with high-level facial structure. Moreover, using several statistics along with VLAD for aggregating the extracted features further increases the discriminataive power of our proposed approach.

\section{CONCLUSION}

Automatic pain assessment is important in disease diagnosis applications. This paper introduced a novel framework to represent the facial expression variations for robust pain intensity recognition. Our proposed method partitions a given video into several non-overlapping segments and randomly selects snippets of frames from each segment. These snippets are fed into a CNN for feature extraction. We incorporated low-level visual patterns and high-level structural information in an aggregating layer. The obtained features are encoded into a compact binary code using a deep network. The facial expressions are represented by a single binary code so that those which are caused by a same level of pain have smaller Hamming distance. Extensive experiments on the publicly available UNBC-McMaster database demonstrated the effectiveness of our proposed method compared to many existing works.

\section{ACKNOWLEDGEMENT}

The financial support of the Academy of Finland and Infotech Oulu is acknowledged.

\section{REFERENCES}

[1] M. Lynch, "Pain as the fifth vital sign," Journal of Intravenous Nursing, vol. 24, no. 2, pp. 85-94, 2001. 
[2] A. Williams, H. Davies, and Y. Chadury, "Simple pain rating scales hide complex idiosyncratic meanings," Pain, vol. 85, no. 3, pp. 457-463, 2000.

[3] K. M. Prkachin and P. E. Solomon, "The structure, reliability and validity of pain expression: Evidence from patients with shoulder pain," Pain, vol. 139, no. 2, pp. 267-274, 2008.

[4] A. B. Ashraf, S. Lucey, J. F. Cohn, T. Chen, Z. Ambadar, K. M. Prkachin, and P. E. Solomon, "The painful face pain expression recognition using active appearance models," Image and Vision Computing, vol. 27, no. 12, pp. 1788-1796, 2009.

[5] P. Lucey, J. F. Cohn, I. Matthews, S. Lucey, S. Sridharan, J. Howlett, and K. M. Prkachin, "Automatically detecting pain in video through facial action units," IEEE Trans. Systems, Man, and Cybernetics, Part B (Cybernetics), vol. 41, no. 3, pp. 664-674, 2011.

[6] S. Kaltwang, S. Todorovic, and M. Pantic, "Doubly sparse relevance vector machine for continuous facial behavior estimation," IEEE Trans. PAMI, vol. 38, no. 9, pp. 1748-1761, 2016.

[7] C. Florea, L. Florea, and C. Vertan, "Learning pain from emotion: Transferred hot data representation for pain intensity estimation," in ECCV Workshops, 2015, pp. 778-790.

[8] R. Zhao, Q. Gan, S. Wang, and Q. Ji, "Facial expression intensity estimation using ordinal information," in IEEE CVPR, 2016, pp. 3466-3474.

[9] J. Zhou, X. Hong, F. Su, and G. Zhao, "Recurrent convolutional neural network regression for continuous pain intensity estimation in video," in IEEE CVPR Workshops, 2016, pp. 1535-1543.

[10] J. Egede, M. Valstar, and B. Martinez, "Fusing deep learned and hand-crafted features of appearance, shape, and dynamics for automatic pain estimation," in IEEE FG, 2017, pp. 689-696.

[11] H. Jégou, M. Douze, C. Schmid, and P. Pérez, “Aggregating local descriptors into a compact image representation," in IEEE CVPR, 2010, pp. 3304-3311.

[12] Y. Gong, S. Lazebnik, A. Gordo, and F. Perronnin, "Iterative quantization: A procrustean approach to learning binary codes for large-scale image retrieval," IEEE Trans. PAMI, vol. 35, no. 12, pp. 2916-2929, 2013.

[13] W. Liu, J. Wang, R. Ji, Y. G. Jiang, and S. F. Chang, "Supervised hashing with kernels," in IEEE CVPR, 2012, pp. 2074-2081.
[14] P. Lucey, J. F. Cohn, K. M. Prkachin, P. E. Solomon, and I. Matthews, "Painful data: The unbc-mcmaster shoulder pain expression archive database," in IEEE $F G, 2011$, pp. 57-64.

[15] D. Yi, Z. Lei, S. Liao, and S. Z. Li, "Learning face representation from scratch,” arXiv, vol. 1411.7923, 2014.

[16] S. Kaltwang, O. Rudovic, and M. Pantic, "Continuous pain intensity estimation from facial expressions," in Advances in Visual Computing, 2012, pp. 368-377.

[17] P. Rodriguez, G. Cucurull, J. Gonzàlez, J. M. Gonfaus, K. Nasrollahi, T. B. Moeslund, and F. X. Roca, "Deep pain: Exploiting long short-term memory networks for facial expression classification," IEEE Trans. Cybernetics, vol. PP, no. 99, pp. 1-11, 2018. 